\title{
AN APPLICATION OF KINECT-BASED 3D SCANNING IN BIOMEDICAL ENGINEERING
}

\author{
Octavian Ciobanu1 ${ }^{*}$, Gabriela Ciobanu²
}

${ }_{1}$ "Grigore T. Popa” University of Medicine and Pharmacy, Faculty of Medical Bioengineering, Iasi, Romania ${ }_{2}$ "Gheorghe Asachi” Technical University of Iasi, Faculty of Chemical Engineering and Environmental Protection, Iasi, Romania

\begin{abstract}
This paper approaches the study on the use of a low-cost Kinect sensor for $3 D$ scans and $3 D$ reconstructions of human surfaces. The Kinect depth sensor used in this work is based on a webcam-style device and dedicated software. It uses a structured light technique in order to scan and reconstruct $3 D$ surfaces. The target surface was the human head and the aim was to manufacture an orthotic mask for patients with traumatic burn injuries of the face.

Method: The study was made in order to observe the applicability of the Kinect-based $3 D$ scans in the case of anatomic surfaces with concavities. Anatomic surfaces of a human head were scanned and $3 D$-reconstructed using the Kinect sensor and the Skanect software. Following some $3 D$ image processing, a customized acrylic face mask was fabricated from the $3 D$-reconstructed mesh of the head. Orthotic masks may be applied in the case of facial burns in order to provide a small pressure over the burns. Results: The study shows the applicability of the Kinect-based $3 D$ scans in the case of anatomic surfaces with rounded concavities and especially in the case of patients with burn injuries of the face. This study is among the first approaches to the application of the scanning of burn injuries with a Kinect device.
\end{abstract}

Key words: $3 D$ scanning, Kinect, orthotic mask, bioengineering, application

DOI: $10.21175 /$ RadProc.2016.43

\section{INTRODUCTION}

Digital cameras, laser scanning devices, structured light scanning devices and other optical sensors have numerous biomedical applications. The structured light scanning technique is becoming one of the most cost-effective solutions for the $3 \mathrm{D}$ scanning of anatomic surfaces and small objects. Compared to other scanning systems, the main advantages of depth sensors (like Kinect, Asus Xtion, etc) based on structured light, is that the devices are webcam-style peripheral for PC as well as have low prices, between 200 and 1000 Euros.

The Kinect device is a multisensory peripheral for Microsoft Xbox gaming console. The depth sensor is used for measuring the three-dimensional coordinates of the points of a surface, using projected light patterns and a camera system. The depth camera system processes live data of a multitude of coordinates into a reconstructed $3 \mathrm{D}$ model in real-time.

The Kinect depth sensor uses a structured light technique in order to reconstruct virtual $3 \mathrm{D}$ surfaces described by point clouds defined by three dimensional coordinates. The depth sensor allows the users to make scans around any object with a radius between 0.4 and 1 meter.

Paper approaches the case of severe burns around the face, when a customized acrylic face mask must be fabricated and applied in order to provide a small pressure over the burns.

Facial burn treatments involve removing the damaged tissue followed by grafting and other recovery procedures. The main objectives in burn tissue recovery are functions restoration, comfort and skin aesthetics. Hypertrophic scarring, Keloid scarring and scar contracture, are some of the major outcomes of a thermal injury. The application of an orthotic mask may improve the rehabilitation of burns [1], [2]. Pressure applied to a scar decreases the excessive collagen accumulation and the formation of scars.

3D scanning has a lot of medical and bioengineering applications: anthropometry, aesthetic surgery, aerospace medicine, human recognition, dentistry, prosthetics, orthotics, posture evaluation, rehabilitation, ergonomics, forensics etc. [3]-[6].

The study was made in order to observe the applicability of the Kinect based 3D scans in the case of anatomic surfaces with concavities. The surfaces of the face of a human head were scanned and $3 \mathrm{D}$ reconstructed using the Kinect depth sensor and the Skanect software.

\section{MATERIALS AND METHODS}

In this paper, we explore the use of a depth camera in the obtaining of a 3D model of a face mask for burns.

\footnotetext{
*oct.ciobanu@ gmail.com
} 
There are described the phases from data acquisition, to $3 \mathrm{D}$ reconstruction of the anatomic surface and model mask preparation for $3 \mathrm{D}$ printing.

The Kinect depth sensor is an input device for Microsoft Xbox gaming console. It is used for measuring the three-dimensional coordinates of the points of a surface (point clouds) of an object, using projected light patterns and a camera system (see Fig. 1). The Kinect sensor uses a structured light technique in order to reconstruct $3 \mathrm{D}$ surfaces described by a multitude of point clouds defined by $\mathrm{x}, \mathrm{y}$ and $\mathrm{z}$ coordinates. The depth sensor allows the user to rotate around the object with a radius between 400 and 1000 $\mathrm{mm}$.

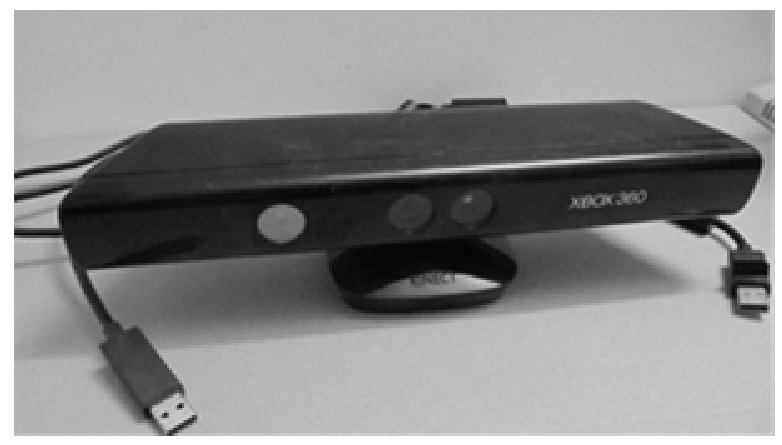

Figure 1. Kinect Xbox 360

Shin and all [6] had studied the accuracy of the Kinect sensor and found in 2013 that the reconstruction errors were between 2 and $3 \mathrm{~mm}$ for human surfaces when sensor was placed at 500 and $2000 \mathrm{~mm}$ distance. These errors allow the use of Kinect sensor in a lot of bioengineering applications including prosthetics and orthotics.

Surface geometry reconstruction using depth sensors is a well-studied area of research in computer graphics. The Kinect for Xbox 360 sensors and the Kinect for Windows sensors have a lot of medical applications [7]-[9].

As the operator and camera describe a trajectory in the space around the target object during scanning, data of the object are processed on line. Using trigonometric triangulation, the scanning system calculates the coordinates of the point cloud of object. The device consists of an infrared projector, an infrared depth camera, a RGB camera, a multi-array microphone and a three-axis accelerometer. It weighs about $0.5 \mathrm{~kg}$.

Depth cameras like the Microsoft Kinect, Asus Xtion and Primesense Carmine have similar characteristics. The Kinect sensor has a projector with a special lens that forms a known pattern of infrared light. The infrared light makes dots on the surfaces of objects it touches visible only with night vision devices as in Fig. 2.

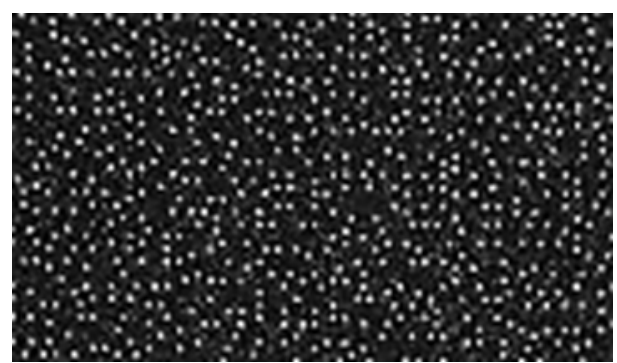

Figure 2. Infrared dots projected by Kinect

The Kinect sensor works with Windows Software Development Kit (SDK). For faster 3D reconstruction, a PC needs next requirements: minimum 2 GB RAM, Windows 7 or later (64 bits), NVidia GTX 560 or higher.

Also, Kinect needs OpenNI, open-source software that process $3 \mathrm{D}$ data from Kinect, and from other sensors. Software and hardware enables users to create other applications that include gesture and voice recognition. There are several software for $3 \mathrm{D}$ reconstruction: Skanect, KScan 3D, Fablitec, ReconstructMe, Blender, etc. Skanect was preferred for this application.

\section{RESUlTS AND DisCUSSION}

Computer Aided Design/Computer Aided Manufacturing (CAD/CAM) and Rapid Prototyping are indispensable tools for the technological improvement in the conception and manufacturing of customized prostheses and orthoses. In order to study the feasibility of the scanning and $3 \mathrm{D}$ reconstruction using Kinect, sensor, a 3D scanning was performed around the head of a healthy person. The trajectory of the Kinect during scanning is presented in Fig. 3.

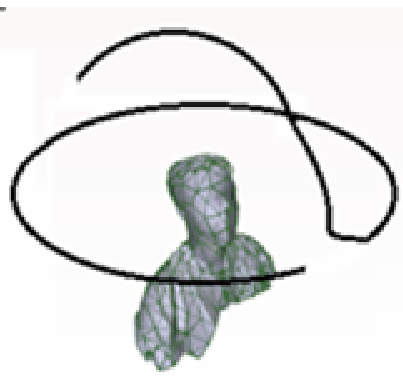

Figure 3. Example of Kinect sensor trajectory during scanning

The quality of $3 \mathrm{D}$ model depends on reconstruction software and on correct scanning process. The $3 \mathrm{D}$ models were obtained through $3 \mathrm{D}$ reconstruction with Skanect software with different densities of polygons. The $3 \mathrm{D}$ model is reconstructed using surface modelling (see Fig. 4) 

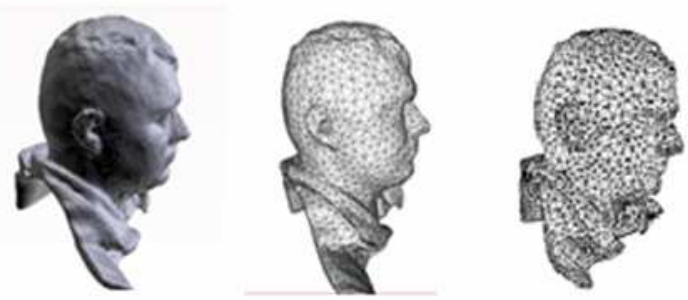

Figure 4. Results of scanning and 3D reconstruction with different densities of polygons

Skanect software used in this study performs $3 \mathrm{D}$ reconstruction, modelling surfaces through several phases. The number of surface polygons may be controlled using free $3 \mathrm{D}$ software like Rhinoceros or MeshLab.

In the initial phase of $3 \mathrm{D}$ reconstruction, Skanect produces a surface model, empty inside. The sectioned surface was obtained with Deep Exploration software (Fig. 5).

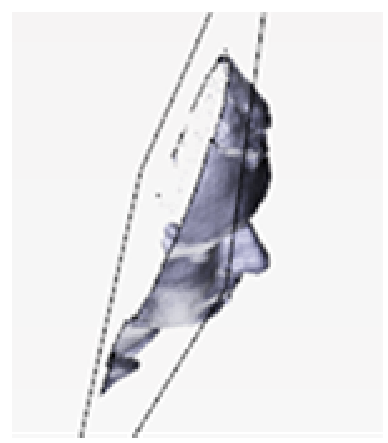

Figure 5. The 3D model of the head, empty inside

After the final processing phase with Skanect, the inner parts of the reconstructed model are filled with polygons. The inner parts of the model are viewed with Deep Exploration software through sectioning (see Fig. $6)$.

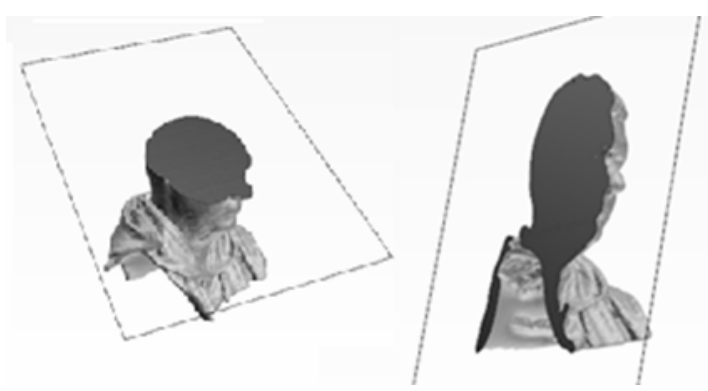

Figure 6. Horizontal and vertical sections performed with Deep Exploration software

The reconstructed $3 \mathrm{D}$ model of the head is useful for the next processing phase in order to obtain the customized surface of future orthotic mask.

In order to study the feasibility of scanning and reconstruction, conventionally only a small portion of the face surface was considered burned as in Fig. 7 .

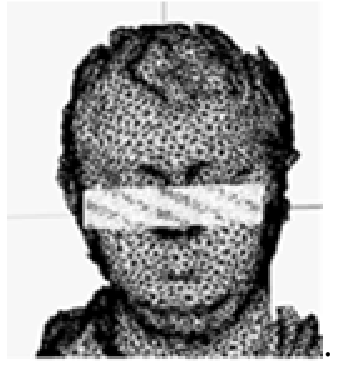

Figure 7. The chosen surface to be protected by an orhotic mask

A $3 \mathrm{D}$ mesh box, covering the chosen area of the face, is superimposed over the $3 \mathrm{D}$ model of the face. Both models have to be modeled as $3 \mathrm{D}$ meshes (see Fig.8).

The scanning was performed in day light, when lighting conditions were stable. The sensor avoids the contact with the patient skin and the scanning takes only 10-15 minutes. In the case of patients with severe facial burns and having reclined head, it is sufficient to scan only for $180^{\circ}$ around the head. The final $3 \mathrm{D}$ model will reconstruct the $3 \mathrm{D}$ mesh of the frontal part of the head, sufficient for obtaining the 3D model of the facial orthosis. Next step is surface mask extraction using superimposing procedure and using CAD software as in Fig. 8.

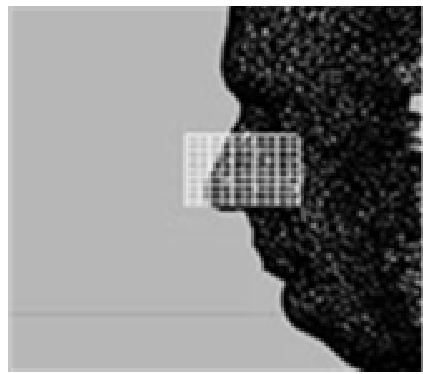

Figure 8. Superimposing a mesh box over the $3 \mathrm{D}$ mesh of face mesh box

The final sectioning (splitting) operation was performed with Rhino 5 software. Mesh Split function of Rhino 5 was extracted from Mesh Edit Tools and from Mesh list. The result of mesh splitting operation is a thin surface.

The visualization of the final surface is made in "shade" mode in order to see all concavities (see Fig. 9).

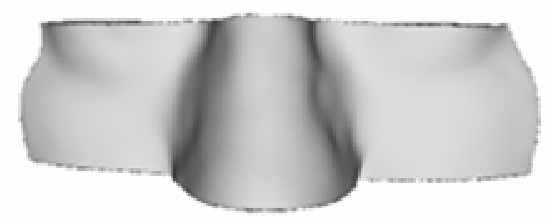

Figure 9. The face mask surface after splitting the face mesh with a mesh box

Finally, the 3D model of the orthotic mask has to be saved in STL format, useful in Rapid Prototyping or 3D 
printing. The fabrication may be accomplished through a professional company for $3 \mathrm{D}$ printing.

Analyzing the $3 \mathrm{D}$ model of the face, most critical surfaces with concavities are placed at nose, at the corner of the eyes, at the intersection line between nose and face and in the inner shapes of the ear. Some of these details of the face are well reconstructed: the corner of the eyes and the intersection line between nose and face. The inner forms of the nose and the inner shapes of the ear are approximate. This means that in the case of an orthotic mask for patients with burn injuries of the face, the pressure on the inner forms of nose and ear will be improperly and it is possible to remain scars during recovery of patients.

The results of the facial reconstruction with Kinect are better comparing to the results obtained with a photogrammetric technique [10]. The photogrammetric technique has difficulties when reconstructing some of face concavities [11]. The visual comparison of the finesse of details of the reconstructed faces show that Kinect based scanning models have better surface details, especially around the eyes, and the intersection lines between nose and face.

\section{CONCLUSION}

This study shows some characteristics of the use of the Kinect depth sensor in the scanning of the face. The sensor avoids the contact with the patient skin during scanning. Also the results of the study show that the Kinect based 3D scans have good performances in the case of face concavities excepting some peculiar parts.

Especially the details around eyes and nose are well reconstructed. However the inner forms of the nose and the inner shapes of the ear are approximate.

As a result, the orthotic masks obtained with Kinect will produce no pressure on the inner forms of nose and ear and it is possible to remain scars during recovery of patients.

Results of the scanning and reconstruction study showed that the Kinect depth camera and reconstruction software yield good $3 \mathrm{D}$ meshes of the patient face which are acceptable for orthotic purposes.

The main advantage of the proposed scanning system is the price: it is about ten times cheaper than a middle class professional scanning device.

\section{REFERENCES}

1. P. Richard, A.C. Masquelet, M.P. Vazquez and C. Accart, "Techniques de prevention par masque facial des cicatrices-hypertrophiques de la face, secondaires aux brulures profondes," Rev Stom Chir Maxillo-Faciale, vol. 87, no. 6, pp. 398-401, 1986 (P. Richard, A.C. Masquelet, M.P. Vazquez and C. Accart, "Techniques for the Prevention of Facemask Hypertrophic Scars - The Opposite Side of the Deep Burns," Rev Stom Chir Maxillo-Faciale, vol. 87, no. 6, pp. 398-401, 1986)

2. M.J. Pilley, C. Hitchens, G. Rose, S. Alexander, and D.I. Wimpenny, "The Use of Non-Contact Structured Light Scanning in Burns Pressure Splint Construction," Burns, vol. 37, no. 7, pp. 1168-1173, Nov. 2011

3. E. Hierholzer and B. Drerup, "High-Resolution Rasterstereography," in: Three-Dimensional Analysis of Spinal Deformities, M. D’Amico, A. Merolli, G.C. Santambrogio, Eds., Amsterdam, Nederlands: IOS Press, ch. 6 , pp. $435-439,1995$

4. T. Jiao, F. Zhang, X. Huang and C. Wang, "Design and Fabrication of Auricular Prostheses by CAD/CAM System," Int. J. Prosthod., vol. 17, no. 4, pp. 460-463, July-Aug. 2004

5. G. Sansoni, M. Trebeschi, G. Cavagnini and G. Gastaldi, "3D Imaging Acquisition, Modeling and Prototyping for Facial Defects Reconstruction," in Proc. SPIE: Thr.-Dim. Imag. Metrol., vol. 7239, pp. 1-8, Jan. 19, 2009

6. B. Shin, R. Venkatramani, P. Borker, A. Olch, J. Grimm and K. Wong, "Spatial Accuracy of a Low Cost High Resolution 3D Surface Imaging Device for Medical Applications," Int. J. Med. Phys., Clin. Engin. Rad. Oncol., vol. 2, no. 2, pp. 45-51, May 2013

7. Y. Li, "Hand Gesture Recognition Using Kinect," in Proc. Int. Conf. Comp. Sci. Autom. Engin., June 2012, pp. 196-199

8. S. Izadi et al., "KinectFusion: Real-Time $3 \mathrm{D}$ Reconstruction and Interaction Using A Moving Depth Camera," in Proc. ACM Symp. UIST, Santa Barbara (CA), USA, 2011, pp. 559-568.

9. I. Oikonomidis, N. Kyriazis, and A. Argyros, "Efficient Model-Based 3D Tracking of Hand Articulations Using Kinect," in Proc. BMVC, Dundee, Scotland, UK, Sep. 2011, pp. 101.1-101.11

10. O. Ciobanu and G. Ciobanu, "A Study on Two Cost Effective Scanning and Modeling Techniques Used for the Fabrication of an Orthotic Mask," Buletinul I.P.I. Secţ. Constr. Mas.., Fasc., vol. 59, no. 4, pp. 1-8, Oct. 2013

11. O. Ciobanu, W. Xu, G. Ciobanu, "An Evaluation of Singlecamera Stereophotogrammetric Scanning Technique Applied in 3D Modeling", Fiabil. Durabil., Suppl. 1,no. 1, pp. 234-240, Jan. 2013 